\title{
Enfermedad hepática y trasplante durante la pandemia COVID-19/SARS-CoV-2
}

\author{
Liver disease and transplantation during \\ the COVID-19/SARS-CoV-2 pandemic
}

\author{
Pilar Leal-Leyte, * Daniel Zamora-Valdés, * Nahum Méndez-Sánchez ${ }^{\ddagger}$ \\ * Grupo de Estudio de Trasplante Hepático en México, Hospital Ángeles Acoxpa. Ciudad de México, México. \\ ₹ Unidad de Hígado, Fundación Clínica Médica Sur. Ciudad de México, México.
}

\section{RESUMEN}

Introducción: La pandemia por SARS-CoV-2/COVID-19 ha afectado todas las áreas de la atención médica, particularmente la atención de enfermedades crónicas. La enfermedad hepática crónica es altamente prevalente en nuestro país y representa una de las principales causas de mortalidad general. Objetivo: En esta revisión, analizamos la literatura disponible hasta el 5 de junio de 2020 sobre el impacto de SARS-CoV-2/COVID-19 en enfermedades hepáticas y en la actividad de trasplante hepático. Material y métodos: Realizamos una búsqueda en PubMed con los términos «liver» (hígado), «hepatic» (hepático), o «transplant» (trasplante) $\mathrm{y}$ «COVID-19» 0 «SARS-CoV-2» contenidos en el título o el resumen de la categoría «Journal Articles» durante el último año. Los artículos fueron analizados por dos revisores de forma independiente. Resultados: Encontramos 276 resultados, de los cuales 54 artículos cumplieron con los criterios de inclusión. La mayor parte de la información disponible proviene de recomendaciones de expertos o consensos. Los organismos reguladores de donación y trasplante han desarrollado algoritmos específicos para su región con el objetivo de guiar la actividad de trasplantes durante la pandemia. Los programas, en general, han reportado disminución en sus actividades. Los resultados de infección por SARS-CoV-2 en receptores de trasplante hepático no son concluyentes y la evaluación del donante para la infección es ampliamente recomendada. Conclusión: La pandemia por SARS-CoV-2/COVID-19 ha modificado las prácticas de todos los programas de trasplante alrededor del mundo. La comunidad de trasplantes se encuentra frente a un escenario difícil con evidencia científica escasa que guíe la toma de decisiones sobre el futuro y la atención óptima de pacientes con enfermedad hepática terminal en necesidad de trasplante, durante y después de la pandemia.

Palabras clave: Hígado, enfermedades hepáticas, trasplante, COVID-19, SARS-CoV-2.

\section{ABSTRACT}

Introduction: SARS-CoV-2/COVID-19 pandemic has affected all healthcare areas, particularly chronic diseases. Chronic liver diseases are highly prevalent in our country and represent a major cause of overall mortality. Objective: In this invited review, we analyze the literature available at June 5, 2020 on the impact of the pandemic on liver disease and transplantation. Material and methods: We performed a PubMed search using the terms «liver», «hepatic» or «transplant» and «COVID-19" or «SARS-CoV-2" included in the title and/or abstract in the category of «Journal Articles» during the last year up to June 5, 2020. The articles were analyzed by two reviewers independently. Results: We found 276 results (abstracts), from which, 54 full manuscripts fulfilled the selection criteria. Most of the available data comes from expert recommendations or consensus. Organ donation and transplantation regulatory bodies have created algorithms to guide transplant activity during the pandemic. Donor testing for SARS-CoV-2 is highly recommended but has several weaknesses. Programs have reported diminished activity during this period. Outcomes on liver transplant recipients with COVID-19 infection are heterogenous. Conclusion: SARSCoV-2/COVID-19 pandemic has forced transplant programs to modify their practices. The transplant community faces a difficult scenario with scant scientific evidence to guide its way and decision making in the treatment of patients with liver failure in need of a lifesaving transplant during and after the pandemic.

Keywords: Liver, liver disease, transplant, COVID-19, SARS-CoV-2. 


\section{INTRODUCCIÓN}

La Organización Mundial de la Salud (OMS) declaró a la enfermedad causada por el coronavirus SARSCoV-2, denominada COVID-19, una emergencia de salud pública internacional el 30 de enero, y como pandemia, el 11 de marzo de 2020. Desde entonces, cada país ha visto afectado su sistema de salud en forma distinta, lo cual ha tenido un gran impacto en el cuidado y atención de pacientes con patologías no urgentes. Sin embargo, ésta no es la primera vez que la comunidad de trasplantes se enfrenta a virus emergentes, pues ya se han tenido experiencias previas con el virus del síndrome respiratorio agudo grave (SARS, por sus siglas en inglés) y el virus respiratorio del Medio Oriente (MERS, por sus siglas en inglés).

Alrededor del mundo, la mayoría de los centros y organismos reguladores en materia de donación y trasplantes ha creado protocolos y medidas para mitigar el impacto que la pandemia tendrá en su área. Dicho esto, ha resultado importante un balance cauteloso frente a la situación incierta ante la cual se encuentra toda la comunidad médica, así como el riesgo de no emplear procedimientos que salvan vidas, tal como lo es el trasplante hepático. En esta revisión sistemática, evaluamos y sintetizamos la literatura relacionada al día 5 de junio de 2020 sobre las manifestaciones hepáticas de la infección por el virus SARS-CoV-2 en pacientes sanos, su impacto en pacientes cirróticos y postrasplante hepático y el impacto de la pandemia en la actividad de trasplante hepático.

\section{MATERIAL Y MÉTODOS}

Se realizó una revisión de la literatura científica dirigida a evaluar el impacto de la pandemia por COVID-19. Los estudios fueron identificados mediante estrategias sensibles de búsqueda bibliográfica en la base de datos PubMed. Los términos utilizados para la captura fueron: «liver», "hepatic» 0 «transplant» y «COVID-19» 0 «SARS-CoV-2». La base de datos Medline fue cribada desde el 5 de junio de 2019 al 5 de junio de 2020. No hubo restricciones de idioma para la revisión de abstracts, pero sólo los manuscritos en inglés, castellano, francés o italiano fueron revisados en extenso. Todas las referencias recuperadas fueron manejadas en EndNote (Thomson Reuters). Finalmente, se realizó una búsqueda manual mediante la revisión de las referencias de los estudios incluidos.

Los estudios recuperados mediante la estrategia anterior de búsqueda fueron incorporados si cumplían con los siguientes criterios de inclusión: que el estudio reportara información relevante original de, al menos, un caso clínico, o bien la experiencia de un centro de trasplante durante la pandemia. Se incluyeron todo tipo de artículos. Se excluyeron estudios en animales y de ciencia básica. Las revisiones sistemáticas y metaanálisis fueron seleccionadas para la revisión en los temas en los que estuvieron disponibles. Dos revisores examinaron los títulos y los resúmenes de los artículos recuperados según los criterios de selección de forma independiente. En caso de no tener resumen, los mismos revisores estudiaron el manuscrito en extenso. Ambos revisores recolectaron los datos de los artículos incluidos, utilizando para ello formularios normalizados ad hoc.

Toda la recolección de artículos fue doble e independiente. Si al hacer esto el revisor encontraba alguna discrepancia entre su información y la del otro revisor, entonces se llegaba a un consenso revisando el artículo original. Los artículos que no contenían información relevante para los objetivos de la revisión o que tenían datos insuficientes fueron excluidos. No hubo metaanálisis planeado entre los estudios incluidos. Identificamos 276 resúmenes en PubMed, de los cuales se seleccionaron 54 , siguiendo los criterios de selección que formaron la base de esta revisión. Para dar contexto a la información y debido a la relevancia de su contenido, 24 referencias más fueron analizadas, incluyendo dos no indizadas en PubMed, las cuales se publicaron en una plataforma de publicación temprana: bioRxiv.

\section{ALTERACIONES HEPÁTICAS EN PACIENTES INFECTADOS POR COVID-19/SARS-COV-2}

La infección por SARS-CoV-2 ataca principalmente el aparato respiratorio. Sin embargo, se ha demostrado que los pacientes con COVID-19 presentan alteraciones a otros niveles. Por ejemplo, la infección puede causar alteraciones en las pruebas de función hepática de forma primaria o secundaria. EI SARS-CoV-2 utiliza a la enzima convertidora de angiotensina-2 como sitio de anclaje para su entrada en las células. ${ }^{1}$ El receptor ACE2 está presente en las células del epitelio biliar. ${ }^{2}$ Además, la expresión de ACE2 en colangiocitos humanos es preservada en organoides hepáticos humanos, modelo experimental en el que se ha demostrado la replicación del virus SARS-CoV-2. ${ }^{3}$

La afección hepática primaria se manifiesta con la elevación de enzimas hepáticas al ingreso. La afección que se genera de forma secundaria a la enferme- 
dad COVID-19 es más común y es consecuencia del estado de inflamación sistémica y choque circulatorio, lo cual genera hipoxia e isquemia hepática. A esto hay que sumar el daño hepático asociado con otros fármacos, que se puede presentar por la polifarmacia en el tratamiento de estos pacientes.

Siete revisiones sistemáticas con metaanálisis publicadas entre el 9 y el 28 de mayo de 2020 analizaron las manifestaciones hepáticas de COVID-19 al ingreso de los pacientes. ${ }^{4-10}$ Kukla y colaboradores analizaron el impacto de tres Coronaviridae en 43 estudios (con presencia de SARS-CoV-1 en 23 estudios y 1,894 pacientes; MERS en nueve estudios y 156 pacientes, y SARS-CoV-2 en estudios y 2,541 pacientes), encontrando alteraciones hepáticas en las tres enfermedades virales con hallazgos histopatológicos inespecíficos. ${ }^{5}$ A diferencia de SARS-CoV-1 y 2 , el MERS requiere de la enzima dipeptidil peptidasa-4 como receptor funcional, y hasta el momento no hay evidencia de que la infección cause daño hepático directo. El hallazgo común de los autores en las tres entidades fue la posibilidad de sesgo en la interpretación de los resultados por la presencia de comorbilidades en los pacientes y el impacto de la respuesta inflamatoria y/o estado de choque durante su estancia en el hospital. El resto de las revisiones sistemáticas se centraron en la infección por SARS-CoV-2.

Sultan y su equipo de investigación revisaron 47 estudios en los que incluyeron a 10,890 pacientes; la incidencia de aspartato aminotransferasa (AST) y del alanino aminotransferasa (ALT) anormales al ingreso fue de $15 \% .{ }^{10}$ Por su parte, Wang y otros revisaron 21 estudios (3,024 pacientes), donde de 2.6 a $53 \%$ tuvo enzimas hepáticas (AST e ALT) anormales. ${ }^{7}$ Tanto Parohan y colaboradores como Youssef y su equipo de investigación realizaron sendos metaanálisis independientes con la información de 20 estudios retrospectivos (3,248 pacientes). Ambos metaanálisis demostraron que los pacientes con hipoalbuminemia, elevación de AST, ALT y bilirrubina total cursaron con enfermedad grave en mayor frecuencia. ${ }^{4,8}$ Mao y su equipo analizaron 35 estudios, incluyendo 6,686 pacientes, donde la prevalencia acumulada de alteraciones hepáticas en pacientes infectados por SARSCoV-2 fue de $19 \%$. Su metaanálisis demostró que la incidencia de alteraciones hepáticas es más común en pacientes graves, particularmente entre los pacientes en Wuhan, China. ${ }^{6}$ El metaanálisis de Kunutsor y colaboradores (16 estudios y 10,540 pacientes, todos de China) demostró que el nivel de AST y ALT al ingreso predijo enfermedad grave (nueve estudios), mientras que AST se asoció con una mayor mortalidad en un metaanálisis de sólo tres estudios. ${ }^{9}$ En un solo estudio, los niveles de fosfatasa alcalina y GGT al ingreso predijeron la mortalidad. ${ }^{11}$

Hay varios problemas con la interpretación de la información disponible a la fecha. Por ejemplo, el nivel de elevación de enzimas, considerado en varios artículos como «disfunción hepática», no corresponde con la definición recomendada para la lesión hepática aguda $(A L T \geq 200 \mathrm{UI} / \mathrm{L}$ y/o AST $\geq 300 \mathrm{UI} / \mathrm{L}) .{ }^{12}$ La diferencia entre los niveles de AST y ALT en pacientes graves y no graves no es clínicamente relevante (AST $8.84 \mathrm{UI} / \mathrm{L}$ y ALT $7.35 \mathrm{UI} / \mathrm{L}$ ) y la incidencia de niveles anormales es altamente variable. ${ }^{4}$ Pocos estudios reportan la prevalencia de enfermedades hepáticas previamente existentes en los pacientes. ${ }^{10}$ La mayoría de los estudios no reporta los valores de la razón normalizada internacional (INR), que en estos pacientes representarían el mejor indicador de lesión hepática aguda grave (INR $\geq$ 2.0)..$^{13}$ Un estudio dedicado a evaluar alteraciones en la coagulación reportó que los pacientes graves con COVID-19 tuvieron un INR promedio de 1.04, y no se encontraron diferencias significativas en comparación con enfermos no graves (INR 1.01). ${ }^{14}$

Los hallazgos histológicos no son específicos, ya que pueden ser explicados por la respuesta inflamatoria sistémica y el estado de choque. ${ }^{15}$ La información disponible que asocia estas alteraciones con gravedad o mortalidad tiene resultados heterogéneos y pacientes/estudios duplicados. De momento, no hay evidencia de que los pacientes con alteraciones hepáticas requieran intervenciones dirigidas específicamente a tratarlas. Si bien, las enzimas AST y ALT pueden ser un marcador estadístico predictor de gravedad en pacientes con COVID-19, y es biológicamente posible que el SARS-CoV-2 utilice al ACE2 como medio de entrada para la infección primaria a nivel hepático, no hay evidencia definitiva que demuestre la presencia del virus como causal directa de estas alteraciones. Por lo tanto, no hay evidencia suficiente que demuestre que la presencia de alteraciones en las pruebas de funcionamiento hepático observada presente alguna infección hepática primaria ni una forma específica de disfunción hepatocelular.

\section{INFECCIÓN POR COVID-19/ SARS-COV-2 EN PACIENTES CON ENFERMEDADES HEPÁTICAS}

Los pacientes con enfermedades hepáticas, por definición, son la población en riesgo para presentar com- 
plicaciones mayores ante infecciones. Los pacientes con enfermedad hepática terminal con infecciones graves tienen opciones de tratamiento limitadas, independientemente de la pandemia de COVID-19. Al respecto, Wang y colaboradores reportó que de 0.8 a $11 \%$ de 3,024 pacientes infectados padecía enfermedad hepática crónica. ${ }^{7}$

Qi y su equipo reportaron esto mismo en tres casos en Hubei, China (cuya etiología era hepatitis B, esquistosomiasis y consumo de alcohol); dos pacientes fallecieron y un paciente egresó. ${ }^{16}$ Estos pacientes también fueron incluidos en un estudio multicéntrico (16 hospitales) que reportó el resultado de 21 pacientes cirróticos (escala de Child-Pugh A, 16 pacientes; escala de Child-Pugh B, tres pacientes, y escala de Child-Pugh $C$, dos pacientes), donde la hepatitis $B$ fue la etiología más común $(57.1 \%)$ y dos terceras partes de los pacientes $(66.7 \%)$ padecían otra comorbilidad. Cinco pacientes fallecieron y no hubo diferencias significativas entre la edad o sexo de acuerdo con el estadio de Child-Pugh o puntaje del modelo para enfermedades hepáticas terminales (MELD) de los supervivientes. ${ }^{17}$

Al incluir a pacientes de Occidente, una revisión sistemática con metaanálisis (22 estudios y 5,595 pacientes) reportó una prevalencia de enfermedad hepática en 2 a $3 \%$ de los pacientes infectados por SARSCoV-2, entre los que $57.33 \%$ de éstos cursó con enfermedad grave y $17.65 \%$ falleció. ${ }^{18}$ Aunque este estudio sugiere que la mortalidad observada en pacientes con enfermedades hepáticas supera a la observada en la población general, no se realizó un análisis estadístico univariado ni multivariado.

Moon y colaboradores analizaron la evolución de 152 pacientes con enfermedades hepáticas crónicas (103 cirróticos y 42 no cirróticos) de 21 países (22.4\% con esteatohepatitis no alcohólica [NASH], 19.7\% con alcoholismo, $11.8 \%$ con hepatitis $\mathrm{B}, 10.5 \%$ con hepatitis C y $35.8 \%$ con otros o su combinación), entre los cuales $23.3 \%$ ingresó a Cuidados Intensivos, $17.5 \%$ recibió ventilación mecánica, $4.9 \%$ se sometió a terapia de reemplazo de función renal y $39.8 \%$ falleció (de este último porcentaje, $78.7 \%$ debido a problemas respiratorios y $12.2 \%$ relacionado con problemas del hígado)..$^{19}$ Treinta y seis punto nueve por ciento de los pacientes presentó un nuevo episodio de descompensación, lo cual se asoció con su estadio Child-Pugh basal, además de que se relacionó con mayor riesgo de mortalidad (63.2\% descompensados en comparación con $26.2 \%$ no descompensados). También, $24.3 \%$ de los pacientes que presentó descompensación no tenía síntomas respiratorios al momento de diagnosticarles
COVID-19. ${ }^{19}$ La mortalidad se asoció con el estadio basal de Child-Pugh en pacientes cirróticos (estadio Child-Pugh A en $23.9 \%$, estadio Child-Pugh B en $43.3 \%$ y estadio Child-Pugh C en $63 \%$ ). Los estadios $\mathrm{B}$ y $\mathrm{C}$ de Child-Pugh fueron predictores de mortalidad después de ajustar por comorbilidades; ${ }^{19}$ no se encontró asociación con el puntaje MELD basal.

Singh y colaboradores realizaron una búsqueda de pacientes con COVID-19 en la plataforma TriNETX en Estados Unidos, identificando a 2,780 pacientes, entre los cuales 250 padecían enfermedades hepáticas (50 cirróticos)..$^{20}$ La prevalencia de comorbilidades fue alta (68\% con hipertensión y $48 \%$ con diabetes mellitus), siendo NASH la etiología más frecuente (con 42\%). Los autores realizaron un pareamiento por la propensión 1:1, ajustado al índice de masa corporal, hipertensión, diabetes mellitus, edad, grupo étnico y uso de nicotina. Después del pareamiento, no hubo diferencias en la incidencia de ALT anormal al ingreso entre los pacientes con enfermedades hepáticas (46.1\%) y aquéllos sin enfermedades hepáticas $(50.6 \%) .{ }^{20} \mathrm{La}$ probabilidad de requerir hospitalización fue mayor entre pacientes con enfermedades hepáticas (48 contra $36 \%$, RR 1.3 [1.1, 1.6], RD 12.0\% [3.4\%, 20.6\%]; p $=0.006)$ y el riesgo de mortalidad fue mayor (RR 3.0 $[1.5,6.0], \operatorname{RD} 8.0 \%$ [3.3\%, 12.7\%]; $p=0.001)$, particularmente en los pacientes cirróticos (RR 4.6, IC95\% 2.6-8.3; $p<0.001) .{ }^{20}$

Wang y otros realizaron un metaanálisis con 34 estudios (6,263 pacientes) con el objetivo de analizar el impacto de las comorbilidades crónicas en los pacientes con COVID-19. ${ }^{21}$ Los resultados de este metaanálisis al analizar la asociación entre las enfermedades hepáticas crónicas y la gravedad de la infección (nueve estudios y 3,196 pacientes) demostraron que no existe asociación entre ambas, a diferencia de lo observado con las enfermedades cardiacas y renales agudas y crónicas. ${ }^{21}$ Este hallazgo obtuvo un bajo grado de heterogeneidad entre los estudios analizados.

Algunos autores han alertado en torno al problema que representa la contingencia para los pacientes con enfermedad hepática crónica y los posibles efectos que ésta pudiera tener en su pronóstico a largo plazo, debido a las posibles limitaciones en su acceso al tratamiento y por el potencial incremento o recaída en el consumo de alcohol y otras drogas. ${ }^{22,23}$

Los registros SECURE-Cirrhosis (Vigilancia Epidemiológica del Coronavirus bajo Exclusión de Investigación en Cirrosis, por sus siglas en inglés) y COVIDHEP podrán proveer de información valiosa una vez que recluten un número mayor de pacientes con se- 
guimiento a mayor plazo. ${ }^{24}$ Sin embargo, por el momento, la información es limitada y no hay evidencia definitiva de que las enfermedades hepáticas crónicas sean un factor de riesgo independiente para la infección por SARS-CoV-2. El riesgo de cursar con enfermedad grave, el ingreso a terapia intensiva y el índice de mortalidad podrían ser mayores que la población general y, aunque este hallazgo es inconsistente en la literatura, ya que la asociación no está detectada en el metaanálisis con la mayor cantidad de pacientes, el riesgo podría ser progresivamente mayor de acuerdo con la gravedad de la enfermedad hepática según la clasificación de Child-Pugh, lo cual podría no ser identificado en los estudios disponibles debido al bajo número de pacientes y el bajo nivel de evidencia.

\section{INFECCIÓN POR COVID-19/SARS-COV-2 EN DONADORES DE ÓRGANOS}

El riesgo de transmisión de SARS-CoV-2 a través del trasplante de órganos sólidos se desconoce. A la fecha, no existen reportes que documenten la transmisión del virus por vía hematógena o por trasplante de órgano sólido, aunque sí se ha detectado la presencia del virus en tejido pulmonar y pleural. ${ }^{25,26}$ Además, se ha documentado la presencia de viremia en 1\% (3 de 307 ) de pacientes sintomáticos, ${ }^{27}$ los cuales pueden tener prueba por reacción en cadena de polimerasa (PCR) por hisopado nasal negativo. ${ }^{28}$

A nivel intestinal, las células epiteliales de la mucosa gástrica, duodenal y colónica, expresan ACE2 en mayores niveles que lo observado en el pulmón. . $^{29,30}$ Asimismo, la presencia del virus en biopsias de mucosa gástrica, duodenal y colónica ha sido demostrada, así como en hisopados anales, los cuales pueden persistir positivos después de la resolución de los síntomas y la negativización del PCR en hisopado nasofaríngeo. ${ }^{30}$ Lo anterior ha tenido un gran impacto en las salas de endoscopia y ha llevado a varias sociedades a emitir recomendaciones para la protección del personal de salud durante procedimientos endoscópicos. ${ }^{31,32}$ Por lo tanto, aunque no existen casos reportados, la transmisión de SARS-CoV-2 mediante trasplante de órgano sólido (pulmón e intestino) parece ser biológicamente posible. Si bien ha sido sugerido que SARS-CoV-2 infecte directamente al riñón y al hígado, la evidencia de infección renal proviene de hallazgos por microscopia electrónica, ${ }^{33}$ y se ha demostrado que estos hallazgos podrían ser explicados por factores diferentes al virus. ${ }^{34}$ La evidencia de afectación hepática ya ha sido discutida y es poco concluyente.
Existen diferentes pruebas para la identificación de SARS-CoV-2, cada una con capacidad diagnóstica diferente. ${ }^{35}$ Vale la pena comenzar con una serie de consideraciones sobre la prueba PCR en hisopado nasal. Primero, es necesario reconocer que hay un porcentaje considerable de pacientes infectados asintomáticos, por lo que en ausencia de síntomas y con una prueba negativa, es imposible determinar la sensibilidad de las pruebas en pacientes asintomáticos. ${ }^{36}$ Segundo, algunos pacientes asintomáticos tienen un PCR en hisopado negativo, pero el virus es detectable mediante PCR en otros tejidos, por lo que la técnica podría no ser la determinante en la capacidad de detección de la infección, sino en el patrón de la enfermedad en ese paciente en específico. ${ }^{27}$ Además, la sensibilidad reportada de la prueba PCR es diferente dependiendo del sitio de toma, las muestras nasofaríngeas tienen mayor sensibilidad (63\%) que las muestras orofaríngeas (32\%), mientras que las muestras de lavado broncoalveolar son las más sensibles (93\%). ${ }^{27}$ Sin embargo, en el contexto de donación cadavérica y en medio de una pandemia, hay que evaluar el riesgo y recursos humanos necesarios para la obtención de muestras del tracto respiratorio inferior. Tercero, algunos pacientes con enfermedad pulmonar característica por tomografía tienen PCR en hisopado nasal negativo, el cual se vuelve positivo durante el seguimiento. ${ }^{37}$ Cuarto, el tiempo necesario para realizar un estudio PCR cualitativo puede ser menor a 15 minutos, pero el tiempo desde la toma de la muestra hasta que se obtiene el resultado no depende solamente del tiempo que demora la prueba, sino de la prioridad con la que sea procesada de acuerdo con la alta demanda, particularmente en áreas con alta incidencia de casos, como en este momento lo es nuestro país.

Los estudios de detección de anticuerpos en suero (serología) pueden realizarse mediante cuatro métodos: ensayos de flujo lateral (LFA, por sus siglas en inglés), ensayos inmunoabsorbentes ligados a enzimas (ELISA, por sus siglas en inglés), ensayos de neutralización e inmunoensayos por quimioluminiscencia. ${ }^{38} \mathrm{La}$ característica común de todos ellos es que demuestran exposición previa a SARS-CoV-2, pero no infección activa, ya que los anticuerpos IgM e IgG son detectables de tres a seis días después de la exposición y los anticuerpos dirigidos contra otros coronavirus persisten en la circulación durante años. ${ }^{39}$

Durante la epidemia de SARS en 2002 se desarrolló una herramienta de tamizaje para donantes cadavéricos. ${ }^{40}$ Dicha herramienta ha sido modificada y, 
en la actualidad, se utiliza por varios programas para el tamizaje del donante cadavérico. Esto ha permitido continuar la actividad de donación, pues ayuda a la identificación o descarte oportuno de potenciales donantes. Varios organismos reguladores de diferentes naciones han realizado algoritmos y protocolos de manejo para los donantes, de acuerdo con su riesgo epidemiológico y prueba de tamizaje..$^{41,42}$ La actividad de donación cadavérica ha disminuido en todos los países que han reportado sus estadísticas debido al consumo de recursos dirigidos a la atención de la pandemia, ${ }^{42,43}$ pero esta reducción parece ser variable en los diferentes centros. ${ }^{44,45}$ Los problemas logísticos de las diferentes pruebas deben ser resueltos en cada centro antes de considerar establecer una política de donación cadavérica basada en resultados, aunque como describimos anteriormente, los argumentos para apoyar este abordaje tienen poca evidencia científica. El riesgo de realizar el procedimiento de trasplante debe de ser balanceado con el riesgo de mortalidad del receptor en lista de espera y discutido ampliamente con el paciente y/o su familia.

La actividad en los programas de hígado de donante vivo se ha reducido, ${ }^{46}$ aunque es importante mencionar que esta actividad varía significativamente de acuerdo con la región geográfica en donde se lleve a cabo. La mayoría de los países occidentales han detenido sus programas de donante vivo de hígado durante la pandemia. En algunos países asiáticos, donde la actividad ha continuado, ${ }^{47,48}$ se recomienda realizar la prueba de PCR para SARS-CoV-2 en hisopado nasofaríngeo en todos los casos de donante vivo de hígado. Si la prueba es positiva, se difiere el procedimiento por un periodo variable, que va desde los siete días hasta un mes, y posteriormente, hay que repetir la prueba. En caso de que la prueba sea negativa, el donante se mantiene en aislamiento siete días después de la prueba y antes del procedimiento. El grupo del Centro Médico Asan reportó un caso de trasplante de hígado de donador vivo infectado (no identificado antes de la donación) que no ha generado enfermedad (ni virus detectable) en el receptor. ${ }^{49}$ El donador no mostraba viremia ni virus detectable en la biopsia hepática, pero tuvo un PCR en hisopado nasofaríngeo positivo que se realizó después de la donación cuando se le notificó contacto previo con una paciente con COVID-19. Después de una revisión de las guías nacionales en la India, se retomó la actividad de trasplante hepático de donante vivo. Verma y colaboradores reportaron nueve casos sin infección en donadores ni en receptores por SARS-CoV-2 y sin mortalidad. ${ }^{48}$

\section{IMPACTO DEL COVID-19/SARS-COV-2 EN LOS PROGRAMAS DE TRASPLANTE HEPÁTICO}

En muchos países alrededor del mundo, las cirugías electivas fueron suspendidas durante la pandemia, ${ }^{50}$ debido al riesgo de infección de los pacientes durante el periodo perioperatorio dentro del hospital ${ }^{51} \mathrm{y}$ el riesgo incrementado de mortalidad descrito durante el periodo de incubación (en Wuhan) en pacientes postquirúrgicos que ingresaron a terapia intensiva. ${ }^{52}$

El trasplante hepático es el tratamiento de elección para pacientes con enfermedad hepática crónica terminal, insuficiencia hepática aguda, algunas enfermedades metabólicas y tumores hepáticos seleccionados. A diferencia de la falla de otros órganos (por ejemplo, riñón), las opciones de tratamiento de la función hepática a largo plazo, en ausencia de trasplante, son nulas. Los pacientes que se encuentran en lista de espera tienen una ventana de oportunidad para trasplante finita, aún fuera de una situación extrema, como lo es una pandemia. La realización de este procedimiento se ha considerado prioritaria durante la pandemia en Estados Unidos y otros países (https://www.cms.gov/files/ document/covid-elective-surgery-recommendations. pdf), para lo cual se han desarrollo protocolos que permitan continuar con la actividad de trasplante en forma segura y así poder mitigar el impacto de la pandemia en el tiempo y mortalidad en lista de espera.

Cada centro debe valorar la situación epidemiológica, los recursos con los que cuenta (materiales y humanos) y el riesgo, tanto del donante como del receptor, así como del personal de salud, cuando se decida proceder o no con la realización del trasplante..$^{53}$

Si bien los modelos de manejo alrededor del mundo varían, los programas más importantes del mundo describieron y adoptaron un abordaje escalonado en la disminución de sus actividades. ${ }^{41}$ Muchos países iniciaron con una disminución progresiva y gradual que se ajustó al avance de la pandemia en su región. ${ }^{42,54,55}$ La recomendación de la suspensión total de la actividad de donación y trasplantes es congruente, exclusivamente cuando el sistema de salud se encuentra absolutamente colapsado. ${ }^{41}$

En Estados Unidos, Canadá y Europa, los programas de donación cadavérica, en general, continuaron con mayor libertad que los de donante vivo estableciendo restricciones, como limitar el trasplante a pacientes con determinado MELD (>20, > 25 o > 30), falla hepática aguda, pacientes en quienes se tratara del primer trasplante y se anticipara uso bajo de productos hemáticos, aquéllos que estuvieran en riesgo de salir 
de criterios para trasplante por patología oncológica, o bien pacientes críticamente graves que estuvieran hospitalizados o que ya se encontraran consumiendo recursos médicos de forma significativa. ${ }^{46}$ Varios programas han reportado disminución global en su actividad de trasplante, el cual va de 25 a $30 \%$ al compararla con el promedio de años previos. ${ }^{54,56}$

Los pacientes pediátricos representan un grupo particular durante la pandemia. Aunque la información es muy limitada, la incidencia de infección asintomática en niños parece ser más frecuente que en los adultos; y, aunque la incidencia de neumonía demostrada por estudios de imagen es alta, el requerimiento de oxígeno y la necesidad de ventilación mecánica dista mucho de lo observado en adultos. ${ }^{57,58}$ Eso sugiere que si el riesgo de mortalidad en la lista de espera es mayor al riesgo de las complicaciones mayores generadas por SARS-CoV-2, los programas de trasplante pediátrico deben continuar su actividad siempre y cuando puedan asegurar la seguridad de su personal de salud. ${ }^{59}$

En nuestro país, el Centro Nacional de Trasplantes recomendó suspender temporalmente todos los programas de donación y trasplante de órganos y tejidos a nivel nacional el 17 de marzo de 2020, cuando había 93 casos de COVID-19 a nivel nacional. El primero de abril de 2020, este centro emitió dos algoritmos para continuar con la donación cadavérica para el tratamiento de urgencias nacionales de corazón e hígado, así como la asignación prioritaria de riñones y córneas: uno para reporte de urgencia y otro para reporte de donación, los cuales no se han actualizado hasta la fecha de realización del presente estudio. Durante este periodo, se han reportado casos aislados de trasplante de córnea por asignación prioritaria.

\section{INFECCIÓN POR COVID-19/SARS-COV-2 EN RECEPTORES DE TRASPLANTE HEPÁTICO}

Los receptores de trasplante suelen tener cargas virales mayores cuando cursan con infección por virus de ARN. Esto genera que los receptores frecuentemente se consideren como «superdiseminadores» (superspreaders), por lo que las precauciones de contacto con familiares y el personal de salud deben ser tomadas seriamente. Además de los factores pronósticos habituales, en los receptores de trasplante la temporalidad de la infección con respecto a la realización del trasplante es una variable importante por considerar. Los pacientes recién trasplantados suelen tener grados de inmunosupresión mayores en comparación con pacientes que se encuentran a más de tres meses después del trasplante. ${ }^{60,61}$ Las recomendaciones en torno a la inmunosupresión durante el periodo de la infección siguen los mismos principios que otro tipo de infecciones sistémicas graves. ${ }^{62-64}$ En los pacientes con manifestaciones menores, se recomienda continuar con el esquema de inmunosupresión habitual.

Tabla 1: Resultados de la infección por SARS-CoV-2 en receptores de trasplante hepático.

\begin{tabular}{|c|c|c|c|c|c|c|}
\hline Hammami et al. ${ }^{67}$ & 1 & 63.0 & 10.0 & 0 & 0.0 & 0.0 \\
\hline Zhong et al. ${ }^{68}$ & 1 & 37.0 & 9 días & 0 & 0.0 & 0.0 \\
\hline Tschopp et al. ${ }^{69}$ & 5 & 56.0 & 47 meses & ND & 0.0 & 0.0 \\
\hline Hoek et al. ${ }^{72}$ & 1 & 47.0 & 19.0 & 83 & 0.0 & 0.0 \\
\hline Liu et al. ${ }^{61}$ & 1 & 50.0 & 2.5 & ND & 100 & 0.0 \\
\hline Fung et al. ${ }^{73}$ & 1 & 80.0 & 14.2 & ND & 0.0 & 0.0 \\
\hline Müller et al. ${ }^{74}$ & 1 & 55.0 & 1.0 & 100 & 0.0 & 0.0 \\
\hline Yi et al..$^{75}$ & 3 & 54.8 & 5.5 & 90 & 33.3 & 0.0 \\
\hline Modi et al. ${ }^{76}$ & 1 & 32.0 & 7.0 & 100 & 0.0 & 0.0 \\
\hline
\end{tabular}

$\mathrm{ND}=$ no disponible. ${ }^{*}$ Promedio en series de caso. 
Cuando la infección tiene una manifestación más grave o fallas orgánicas, se recomienda la disminución o suspensión de la inmunosupresión con monitoreo cercano de las pruebas de funcionamiento hepático y el reinicio de acuerdo con la clínica del paciente. Se necesita monitorizar de cerca posibles interacciones entre los medicamentos frecuentemente empleados como tratamiento para COVID-19 (azitromicina, lopinavir, ritonavir, etcétera) ${ }^{65} \mathrm{~A}$ pesar de múltiples comunicaciones, no hay evidencia de que el manejo con dosis mayores de esteroides o tacrolimus mejore el pronóstico de los pacientes graves con COVID-19.

Los reportes de la enfermedad COVID-19 en receptores de trasplante de órgano sólido son altamente heterogéneos. Mientras que algunos reportes y series de casos describen desenlaces similares a los de la población en general, existen también aquéllos con resultados menos favorables. Los resultados de estos reportes se resumen en la Tabla 1. Hasta el día de corte de esta revisión, hay 184 casos de receptores de trasplante hepático infectados por COVID-19 reportados en la literatura. De éstos, 30\% tuvo una forma grave de la enfermedad (definida por la necesidad de ventilación mecánica) y $14 \%$ falleció. La información disponible no permite evaluar el efecto de las comorbilidades como cofactores en el desenlace de la enfermedad, pero las series de casos que reportan la prevalencia de comorbilidades muestran un gran número de pacientes (56-92\%) con estados comórbidos. Sin embargo, se necesita de más información para poder concluir si los receptores de trasplante hepático tienen un pronóstico diferente a la población general tras la infección por SARS-CoV-2.

\section{CONCLUSIONES}

La cantidad de publicaciones durante la pandemia por COVID-19 es abrumadora. No obstante, el nivel de evidencia de la mayoría de éstas es bajo (recomendación de experto, extrapolación de otras entidades, casos clínicos) y existen diferentes medios publicando manuscritos sin revisión por pares o con una revisión abreviada, por considerar a la pandemia como una urgencia de publicación y de necesidad por compartir información. Esto ha llevado a que muchas series de casos e, incluso metaanálisis, sean publicados en forma de cartas al editor. La investigación básica puede acelerarse y multiplicarse según la demanda, pero la investigación clínica no es tan maleable y la obtención de conclusiones rápidas puede llevar a cometer errores importantes con consecuencias graves.
Por ejemplo, es imposible saber en estos momentos el impacto final que tendrá la reducción de la actividad de trasplante hepático en el mundo en la mortalidad de pacientes en lista de espera, ya que además de los pacientes que pierdan la vida durante la pandemia, el rezago en la atención de aquéllos que se mantuvieron estables durante este periodo traerá como consecuencia un incremento en la mortalidad de la lista de espera incluso después de que la pandemia haya pasado, donde uno de los escenarios posibles es que la enfermedad se vuelva endémica y tengamos que adaptar nuestra práctica a esta «nueva realidad».

La pandemia por COVID-19 sin duda será uno de los obstáculos más grandes al que se enfrentará la comunidad de donación y trasplantes en nuestro tiempo, pero es importante reconocer que la forma en la que respondamos a la demanda de conocimiento científico y atención médica definirá nuestra generación.

Los pacientes con enfermedad hepática terminal y sus médicos tratantes deberán tomar decisiones difíciles frente a la evidente escasez de recursos que durará seguramente más que la pandemia, debido a las repercusiones económicas globales que se están generando durante ésta. Cada programa de donación y trasplante debe evaluar y administrar sus recursos, a la vez que promueve la cooperación entre individuos, instituciones y países con el fin de optimizar la atención de pacientes, candidatos y receptores de trasplante.

\section{REFERENCIAS}

1. Yan R, Zhang Y, Li Y et al. Structural basis for the recognition of SARS-CoV-2 by full-length human ACE2. Science. 2020; 367: 1444-1448.

2. Chai $X, \mathrm{Hu} L$, Zhang $Y$ et al. Specific ACE2 expression in cholangiocytes may cause liver damage after 2019-nCoV infection. bioRxiv. 2020; 2020.02.03.931766.

3. Zhao B, Ni C, Gao R et al. Recapitulation of SARS-CoV-2 infection and cholangiocyte damage with human liver ductal organoids. Protein Cell. 2020; 1-5.

4. Parohan M, Yaghoubi S, Seraj A. Liver injury is associated with severe Coronavirus disease 2019 (COVID-19) infection: a systematic review and meta-analysis of retrospective studies. Hepatol Res. 2020.

5. Kukla M, Skonieczna-Żydecka K, Kotfis K et al. COVID-19, MERS and SARS with Concomitant Liver Injury-Systematic Review of the Existing Literature. J Clin Med. 2020; 9 (5): E1420.

6. Mao R, Qiu Y, He JS et al. Manifestations and prognosis of gastrointestinal and liver involvement in patients with COVID-19: a systematic review and meta-analysis. Lancet Gastroenterol Hepatol. 2020.

7. Wang H, Qiu P, Liu J, Wang F, Zhao Q. The liver injury and gastrointestinal symptoms in patients with Coronavirus Disease 19: A systematic review and meta-analysis. Clin Res Hepatol Gastroenterol. 2020. 
8. Youssef M, Hussein M, Attia AS et al. COVID-19 and Liver Dysfunction: a systematic review and meta-analysis of retrospective studies. J Med Virol. 2020.

9. Kunutsor SK, Laukkanen JA. Markers of liver injury and clinical outcomes in COVID-19 patients: A systematic review and metaanalysis. J Infect. 2020.

10. Sultan $S$, Altayar $O$, Siddique SM et al. AGA Institute rapid review of the gastrointestinal and liver manifestations of COVID-19, meta-analysis of international data, and recommendations for the consultative management of patients with COVID-19. Gastroenterology. 2020.

11. Chen $T, W u$ D, Chen $H$ et al. Clinical characteristics of 113 deceased patients with coronavirus disease 2019: retrospective study. BMJ. 2020; 368: m1091.

12. Dufour DR, Lott JA, Nolte FS et al. Diagnosis and monitoring of hepatic injury. II. Recommendations for use of laboratory tests in screening, diagnosis, and monitoring. Clin Chem. 2000; 46: 2050-2068.

13. Koch DG, Speiser JL, Durkalski V et al. The natural history of severe acute liver injury. Am J Gastroenterol. 2017; 112: 1389-1396.

14. Zou Y, Guo H, Zhang Y et al. Analysis of coagulation parameters in patients with COVID-19 in Shanghai, China. Biosci Trends. 2020.

15. Philips CA, Ahamed R, Augustine P. SARS-CoV-2 related liver impairment - perception may not be the reality. J Hepatol. 2020.

16. Qi X, Wang J, Li X et al. Clinical course of COVID-19 in patients with pre-existing decompensated cirrhosis: initial report from China. Hepatol Int. 2020.

17. Qi X, Liu Y, Wang J et al. Clinical course and risk factors for mortality of COVID-19 patients with pre-existing cirrhosis: a multicentre cohort study. Gut. 2020.

18. Oyelade T, Alqahtani J, Canciani G. Prognosis of COVID-19 in patients with liver and kidney diseases: an early systematic review and meta-analysis. Trop Med Infect Dis. 2020; 5.

19. Moon AM, Webb GJ, Aloman $C$ et al. High mortality rates for SARS-CoV-2 infection in patients with pre-existing chronic liver disease and cirrhosis: preliminary results from an international registry. J Hepatol. 2020.

20. Singh S, Khan A. Clinical characteristics and outcomes of COVID-19 among patients with pre-existing liver disease in united states: a multi-center research network study. Gastroenterology. 2020.

21. Wang X, Fang X, Cai Z et al. Comorbid chronic diseases and acute organ injuries are strongly correlated with disease severity and mortality among COVID-19 patients: a systemic review and meta-analysis. Research (Wash D C). 2020; 2020: 2402961.

22. Da BL, Im GY, Schiano TD. COVID-19 hangover: a rising tide of alcohol use disorder and alcohol-associated liver disease. Hepatology 2020.

23. Reddy KR. SARS-CoV-2 and the liver: considerations in hepatitis B and hepatitis C infections. Clin Liver Dis (Hoboken). 2020; 15 : 191-194.

24. Cha MH, Regueiro M, Sandhu DS. Gastrointestinal and hepatic manifestations of COVID-19: A comprehensive review. World J Gastroenterol. 2020; 26: 2323-2332.

25. Schaller T, Hirschbuhl K, Burkhardt $\mathrm{K}$ et al. Postmortem examination of patients with COVID-19. JAMA. 2020.

26. Nunes Duarte-Neto A, de Almeida Monteiro RA, da Silva LFF et al. Pulmonary and systemic involvement of COVID-19 assessed by ultrasound-guided minimally invasive autopsy. Histopathology. 2020

27. Wang $W, X u Y$, Gao $R$ et al. Detection of SARS-CoV-2 in different types of clinical specimens. JAMA. 2020.
28. Zhang $\mathrm{W}, \mathrm{Du} \mathrm{RH}$, Li B et al. Molecular and serological investigation of 2019-nCoV infected patients: implication of multiple shedding routes. Emerg Microbes Infect. 2020; 9: 386389.

29. Du M, Cai G, Chen F et al. Multiomics evaluation of gastrointestinal and other clinical characteristics of COVID-19. Gastroenterology. 2020; 158: 2298-2301.

30. Xiao F, Tang $M$, Zheng $X$ et al. Evidence for gastrointestinal infection of SARS-CoV-2. Gastroenterology. 2020; 158: 18311833.

31. Castro Filho EC, Castro R, Fernandes FF, Pereira G, Perazzo H. Gastrointestinal endoscopy during the COVID-19 pandemic: an updated review of guidelines and statements from international and national societies. Gastrointest Endosc. 2020.

32. Repici A, Pace F, Gabbiadini R et al. Endoscopy units and the COVID-19 outbreak: a multi-center experience from Italy. Gastroenterology. 2020.

33. Abbate M, Rottoli D, Gianatti A. COVID-19 Attacks the Kidney: ultrastructural evidence for the presence of virus in the glomerular epithelium. Nephron. 2020; 1-2.

34. Roufosse C, Curtis E, Moran L et al. Electron microscopic investigations in COVID-19: not all crowns are coronas. Kidney Int. 2020.

35. Fang FC, Naccache SN, Greninger AL. The laboratory diagnosis of COVID-19-- frequently-asked questions. Clin Infect Dis. 2020.

36. Huff HV, Singh A. Asymptomatic transmission during the COVID-19 pandemic and implications for public health strategies. Clin Infect Dis. 2020.

37. Ai T, Yang Z, Hou H et al. Correlation of chest CT and RT-PCR testing in coronavirus disease 2019 (COVID-19) in China: a report of 1014 cases. Radiology. 2020; 200642.

38. Theel ES, Slev P, Wheeler S et al. The role of antibody testing for SARS-CoV-2: is there one? J Clin Microbiol. 2020.

39. Huang AT, Garcia-Carreras B, Hitchings MDT et al. A systematic review of antibody mediated immunity to coronaviruses: antibody kinetics, correlates of protection, and association of antibody responses with severity of disease. medRxiv. 2020; 2020.04.14.20065771.

40. Kumar D, Tellier R, Draker R, Levy G, Humar A. Severe acute respiratory syndrome (SARS) in a liver transplant recipient and guidelines for donor SARS screening. Am J Transplant. 2003; 3: 977-981.

41. Kumar D, Manuel O, Natori $Y$ et al. COVID-19: A global transplant perspective on successfully navigating a pandemic. Am J Transplant. 2020.

42. Dominguez-Gil B, Coll E, Fernandez-Ruiz $\mathrm{M}$ et al. COVID-19 in Spain: transplantation in the midst of the pandemic. Am J Transplant. 2020.

43. Agnes S, Andorno E, Avolio AW et al. Preliminary analysis of the impact of COVID-19 outbreak on italian liver transplant programs. Liver Transpl. 2020.

44. Massoumi H, Rocca J, Frager S, Kinkhabwala M. COVID-19 infection in early post-operative period after liver transplantation. Liver Transpl. 2020.

45. Agopian V, Verna E, Goldberg D. Changes in liver transplant center practice in response to COVID-19: unmasking dramatic center-level variability. Liver Transpl. 2020.

46. Boyarsky BJ, Po-Yu Chiang T, Werbel WA et al. Early impact of COVID-19 on transplant center practices and policies in the United States. Am J Transplant. 2020.

47. Saigal S, Gupta S, Sudhindran $S$ et al. Liver transplantation and COVID-19 (Coronavirus) infection: guidelines of the liver transplant Society of India (LTSI). Hepatol Int. 2020. 
48. Verma S, Aleen Agarwal S, Chikkala BR et al. Living donor liver transplants for sick recipients during COVID-19 pandemic- An experience from a tertiary center in India. Am J Transplant. 2020.

49. Hong HL, Kim SH, Choi DL, Kwon HH. A case of coronavirus disease 2019-infected liver transplant donor. Am J Transplant. 2020.

50. lacobucci G. Covid-19: all non-urgent elective surgery is suspended for at least three months in England. BMJ. 2020; 368: $\mathrm{m} 1106$.

51. Aminian A, Safari S, Razeghian-Jahromi A, Ghorbani M, Delaney CP. COVID-19 Outbreak and surgical practice: unexpected fatality in perioperative period. Ann Surg. 2020.

52. Lei S, Jiang F, Su W et al. Clinical characteristics and outcomes of patients undergoing surgeries during the incubation period of COVID-19 infection. EClinicalMedicine. 2020; 100331.

53. Chew CA, lyer SG, Chieh Kow AW et al. An international multicentre study of protocols for liver transplantation during a pandemic: a case for quadripartite equipoise. J Hepatol. 2020.

54. Moeckli B, Peloso A, Oldani G et al. The swiss approach to the COVID-19 outbreak. Am J Transplant. 2020.

55. Muller X, Tilmans G, Chenevas-Paule $Q$ et al. Strategies for liver transplantation during the SARS CoV-2 outbreak preliminary experience from a single center in France. Am J Transplant. 2020.

56. Angelico R, Trapani S, Manzia TM et al. The COVID-19 outbreak in Italy: Initial implications for organ transplantation programs. Am J Transplant. 2020.

57. Lu X, Zhang L, Du H et al. SARS-CoV-2 infection in children. $\mathrm{N}$ Engl J Med. 2020; 382: 1663-1665.

58. Dong Y, Mo X, Hu Y et al. Epidemiology of COVID-19 among children in China. Pediatrics. 2020; 145.

59. D'Antiga L. Coronaviruses and immunosuppressed patients: the facts during the third epidemic. Liver Transpl. 2020; 26: 832834.

60. Qin J, Wang $\mathrm{H}$, Qin $X$ et al. Perioperative presentation of COVID-19 disease in a liver transplant recipient. Hepatology. 2020.

61. Liu B, Wang $Y$, Zhao $Y$ et al. Successful treatment of severe COVID-19 pneumonia in a liver transplant recipient. Am J Transplant. 2020.

62. Bollipo S, Kapuria D, Rabiee A et al. One world, one pandemic, many guidelines: management of liver diseases during COVID-19. Gut. 2020.

63. Lau G, Ward JW. Synthesis of liver associations recommendations for hepatology and liver transplant care during the COVID-19 pandemic. Clin Liver Dis (Hoboken). 2020; 15: 204-209.

64. Perazzo H, Piedade J, Castro R et al. COVID-19: an overview of worldwide recommendations for management of patients with liver diseases or liver transplantation. Clin Gastroenterol Hepatol. 2020.

65. Xia T, Wang Y. Coronavirus disease 2019 and transplantation: the combination of lopinavir/ritonavir and hydroxychloroquine is responsible for excessive tacrolimus trough level and unfavorable outcome. Am J Transplant. 2020.

66. Morand A, Roquelaure B, Colson $P$ et al. Child with liver transplant recovers from COVID-19 infection. A case report. Arch Pediatr. 2020.

67. Hammami MB, Garibaldi B, Shah P et al. Clinical course of COVID-19 in a liver transplant recipient on hemodialysis and response to tocilizumab therapy: A case report. Am J Transplant. 2020.

68. Zhong Z, Zhang Q, Xia $\mathrm{H}$ et al. Clinical characteristics and immunosuppressant management of coronavirus disease 2019 in solid organ transplant recipients. Am J Transplant. 2020.

69. Tschopp J, L'Huillier AG, Mombelli M et al. First experience of SARS-CoV-2 infections in solid organ transplant recipients in the swiss transplant cohort study. Am J Transplant. 2020.

70. Gao F, Zheng KI, Gu JY, George J, Zheng MH. COVID-19 and liver transplantation: Lessons learned from three reported cases. Transpl Infect Dis. 2020; e13335.

71. Lee BT, Perumalswami PV, Im GY, Florman S, Schiano TD. COVID-19 in liver transplant recipients: an initial experience from the U.S. epicenter. Gastroenterology. 2020.

72. Hoek RAS, Manintveld OC, Betjes MGH et al. Covid-19 in solid organ transplant recipients: a single center experience. Transpl Int. 2020.

73. Fung M, Chiu CY, DeVoe $C$ et al. Clinical outcomes and serologic response in solid organ transplant recipients with COVID-19: a case series from the united states. Am J Transplant. 2020.

74. Muller H, Kniepeiss D, Stauber R et al. Recovery from COVID-19 following hepatitis $C$, human immunodeficiency virus infection and liver transplantation. Am J Transplant. 2020.

75. Yi SG, Rogers AW, Saharia A et al. Early experience with COVID-19 and solid organ transplantation at a US high-volume transplant center. Transplantation. 2020.

76. Modi AR, Koval CE, Taege AJ et al. Coronavirus disease 2019 in an orthotopic liver transplant recipient living with human immunodeficiency virus. Transpl Infect Dis. 2020; e13351.

77. Patrono D, Lupo F, Canta F et al. Outcome of COVID-19 in liver transplant recipients: a preliminary report from northwestern Italy. Transpl Infect Dis. 2020; e13353.

78. Belli LS, Duvoux C, Karam V et al. COVID-19 in liver transplant recipients: preliminary data from the ELITA/ELTR registry. Lancet Gastroenterol Hepatol. 2020.

\author{
Correspondencia: \\ Pilar Leal-Leyte \\ Grupo de Estudio de Trasplante Hepático México, \\ Hospital Ángeles Acoxpa. \\ Calzada de Acoxpa 430, \\ Col. Coapa, Suite 245, 14308, \\ Ciudad de México, México. \\ Teléfono: +525591304632 \\ E-mail: pilarleal@hotmail.com
}

